\title{
THE HOPF BIFURCATION FOR NONLINEAR SEMIGROUPS
}

\author{
BY J. MARSDEN
}

Communicated by M. H. Protter, November 6, 1972

1. Introduction. Several authors, e.g. [2], [9], [10], [12], [17], have shown by perturbation techniques that the Hopf theorem (see [8], [16]) on the development of periodic stable solutions is valid for the Navier-Stokes equations; in particular, solutions near the stable periodic ones remain defined and smooth for all $t \geqq 0$. The principal difficulty is that the Hopf theorem deals with flows of smooth vector fields on finite-dimensional spaces, whereas the Navier-Stokes equations define a flow (or evolution operator) for a nonlinear partial differential operator (actually it is a nonlocal operator).

The aim of this note is to outline a method for overcoming this difficulty which is entirely different in appearance from the perturbation approach. The method depends on invariant manifold theory [6], [7] plus certain smoothness properties of the flow which actually hold for the NavierStokes flow.

While the statements of the results are relatively simple, the proofs are somewhat complicated in their details. They will not be presented in full here, because all the details of the relevant invariant manifold theory have not yet been published (several details on the applications became clear only after conversations with $\mathrm{M}$. Hirsch). We expect to write out these proofs at a later date. Nevertheless, the summary presented here should give a picture of the method and some idea of the proofs. We hope that the nature of the hypotheses allows our formulation to be fairly readily applicable. There are some indications that this is so. (This is based on conversations with $\mathrm{N}$. Kopell who is working on the bifurcation of periodic solutions in certain chemical systems.)

We shall be considering a system of evolution equations of the general form

$$
d x / d t=Y(x), \quad x(0) \text { given, }
$$

where $Y$ is an operator on a suitable function space $\mathscr{H}$ and will eventually depend on a parameter $\mu$. For example, $Y$ may be the Navier-Stokes operator and $\mu$ the Reynolds number. This system is assumed to define unique local solutions $x(t)$ and thereby a flow $F_{t}$ which maps $x(0)$ to $x(t)$.

The key thing we need to know about the flow $F_{t}$ of our system is that, for each fixed $t, F_{t}$ is a $C^{\infty}$ mapping on a suitable Hilbert space $\mathscr{H}\left(F_{t}\right.$ is

AMS (MOS) subject classifications (1970). Primary 76E30, 35L60, 58B10.

Key words and phrases. Nonlinear semigroups, Navier-Stokes equations, loss of stability, bifurcation, center manifold theory. 
only locally defined in general). This can be verified by fairly general criteria and holds, in particular, for the Navier-Stokes equations; here one uses $\mathscr{H}=H^{s}$ divergence free vector fields, i.e., divergence free vector fields on a bounded domain with $L_{2}$ derivatives of order $\leqq s$ (see [3], [5], $[13],[14])$. Actually this is not quite accurate and the Navier-Stokes equations probably do not define a smooth semigroup. However, this can be remedied by passing to Lagrangian coordinates and there the semigroup is smooth; cf. [3]. (Periodic solutions to the Navier-Stokes equation do not appear periodic in Lagrangian coordinates; but this does not destroy the essence of the method-below we shall ignore this complication.)

One could push the analysis further to include the theory of turbulence as proposed by Ruelle-Takens [16] and in particular see that the solutions remain defined and smooth for all $t \geqq 0$ even after turbulence has set in (cf. [10], [17]). But on the other hand, this mechanism of bifurcation is not well understood - in particular it is not known if "actual" turbulence occurs this way (cf. [10]).

See [15], [18] for general background and further references.

2. Some notation and assumptions on the flow $F_{t}$. Fix a (separable) Hilbert space $\mathscr{H}$ and let $F_{t}$ be a nonlinear semigroup on $\mathscr{H}$. By this we mean the following (cf. [1]):

(a) $F_{t}(x)$ is defined on an open subset of

$$
\boldsymbol{R}^{+} \times \mathscr{H}, \quad \boldsymbol{R}^{+}=\{t \in \boldsymbol{R} \mid t \geqq 0\} ;
$$

(b) $F_{t+s}=F_{t} \circ F_{s}$ (where defined);

(c) $F_{t}(x)$ is separately (hence jointly [1]) continuous in $t, x \in \boldsymbol{R}^{+} \times \mathscr{H}$.

We shall make two standing assumptions on the flow. The first of these is

Smoothness Assumption. Assume that for each fixed $t, F_{t}$ is a $C^{\infty}$ map of (an open set in) $\mathscr{H}$ to $\mathscr{H}$.

This is what we mean by a smooth semigroup. Of course we cannot have smoothness in $t$ since, in general, the generator $X$ of $F_{t}$ will only be densely defined and is not a smooth map of $\mathscr{H}$ to $\mathscr{H}$. However it is known [1] that the derivatives $D^{r} F_{t}(x)$ are automatically jointly continuous in $t$ and $x$ using the strong operator topology. For general conditions under which smoothness holds, see [4], [14]. For the Euler equations and the NavierStokes equations, it is valid in Lagrangian coordinates; see [3], [5], [13], [14], [15]. The simplest situation in which one has smoothness is the case in which the generator $Y$ of $F_{t}$ is obtained as $Y=$ (linear generator) + (smooth, everywhere defined operator). This situation was considered by Segal [19]. Despite the fact that the perturbation is like a bounded operator, this circumstance does apply in a number of problems such as nonlinear wave equations, some chemical reactions involving diffusion, etc. 
The second condition is

Continuation Assumption. Let $F_{t}(x)$, for fixed $x$ lie in a bounded set in $\mathscr{H}$ for all $t$ for which $F_{t}(x)$ is defined. Then $F_{t}(x)$ is defined for all $t \geqq 0$.

This merely states that our existence theorem for $F_{t}$ is strong enough to guarantee that the only way an orbit can fail to be defined is if it tends to infinity in a finite time. This assumption is valid for most situations and in particular for the Navier-Stokes equations; see e.g. [3], [15].

Suppose we have a fixed point of $F_{t}$, which we may assume to be $0 \in \mathscr{H}$; i.e., $F_{t}(0)=0$ for all $t \geqq 0$. Letting $D F_{t}$ denote the Fréchet derivative of $F_{t}$ for fixed $t, G_{t}=D F_{t}(0)$ is clearly a linear semigroup on $\mathscr{H}$. Its generator, say $X$, is therefore a densely defined closed linear operator which represents the linearized equations. Our hypotheses below will be concerned with the spectrum of the linear semigroup $G_{t}$, or equivalently, of $X$.

3. Bifurcation to periodic orbits. Our object now is to give a theorem which not only contains the Hopf theorem as a special case (wherein the vector fields involved are smooth and everywhere defined on $\mathscr{H}$ ) but is sufficiently general so as to apply to the case of smooth nonlinear semigroups. Basically, we have replaced smoothness of the generator with the smoothness assumption described above. The plan of the proof is to follow the proof of Hopf's theorem in Ruelle-Takens [16] but replacing, at the appropriate places, bifurcation theorems for vector fields $Y$ by bifurcation theorems on maps, namely the time $t$-map $F_{t}$. This is outlined below.

We summarize our assumptions as follows:

HyPOTHESES ON THE SPECTRUM. Assume we have a family $F_{t}^{\mu}$ of smooth nonlinear semigroups defined for $\mu$ in an interval about $0 \in \boldsymbol{R}$. Suppose $F_{t}^{\mu}(x)$ is smooth in $x$ for fixed $t, u$ and is jointly continuous in $t, x, \mu$. In addition to the requirements in $\$ 2$, assume

(a) 0 is a fixed point for $F_{t}^{\mu}$;

(b) for $\mu<0$, the spectrum of $G_{t}^{\mu}$ is contained in $D=\{z \in C:|z|<1\}$, where $G_{t}^{\mu}=\left.D_{x} F_{t}^{\mu}(x)\right|_{x=0}$;

(c) for $\mu=0$ (resp. $\mu>0)$ the spectrum of $G_{1}^{\mu}$ at the origin has two isolated simple eigenvalues $\lambda(\mu)$ and $\overline{\lambda(\mu)}$ with $|\lambda(\mu)|=1($ resp. $|\lambda(\mu)|>1)$ and the rest of the spectrum is in $D$;

(d) $\left.(d / d t)|\lambda(\mu)|\right|_{\mu=0}>0$ (the eigenvalues move steadily across the unit circle).

(e) 0 is an attracting fixed point for $\mu=0$, i.e., there is a neighborhood $V$ of 0 such that $F_{t}^{0}(x) \rightarrow 0$ as $t \rightarrow+\infty$ for each $x \in V$.

These hypotheses require a few remarks. First of all, the continuity of $F_{t}^{\mu}(x)$ in $t, x, \mu$ can be verified by fairly general assumptions on the generators, as is discussed in [14]. We certainly can assume smoothness only in the variable $x$. Condition (b) means that, for $\mu<0$, the fixed point 0 is strongly attracting (or "stable") in the sense that the linearized equations 
are attracting, i.e., $G_{t}^{\mu}(x) \rightarrow 0$ as $t \rightarrow+\infty$ for each $x$. Assumption (c) means that, at the critical value $\mu=0$, the linearized terms just fail to be attracting. Finally, (e) means that we assume 0 is still attracting for the nonlinear system, but the attraction is due to the higher order (cubic) terms. This last point is discussed in [11] and in [16]. The same sort of assumption-often difficult to check-occurs in perturbation theory (cf. [10]). N. Kopell has found that in some applications, one does not expect (e) to hold and in that case the periodic solutions one gets are not attracting (or stable) for all nearby solutions. Stability of 0 for $\mu=0$ implies, in particular, by the continuation assumption that the nonlinear flow $F_{t}^{0}$ is automatically defined for all $t \geqq 0$ in a neighborhood of 0 .

THEOREM. Under the above hypotheses, there is a fixed neighborhood $U$ of 0 in $\mathscr{H}$ and an $\varepsilon>0$ such that $F_{t}^{\mu}(x)$ is defined for all $t \geqq 0$ for $\mu \in[-\varepsilon, \varepsilon]$ and $x \in V$. There is a one-parameter family of closed orbits for $F_{t}^{\mu}$ for $\mu>0$, one for each $\mu>0$ varying continuously with $\mu$. They are attracting and hence stable. Solutions near them are defined for all $t \geqq 0$.

The idea of the proof is first to apply the results (Remark 5.3 on p. 177) of [16] to the maps $\Phi_{\mu}=F_{1}^{\mu}$ to conclude the existence of a family of invariant 2-dimensional manifolds parametrized by $\mu$ and then to use Theorem 7.2 of [16] on this restricted family of manifolds to conclude the existence of a unique family of attracting invariant circles for the maps $\Phi_{\mu}$.

The proofs of the theorems referred to in [16] are not complete because, first of all, the invariant manifold theorem is needed for a Hilbert space and for maps (not necessarily diffeomorphisms) and this has not yet been published; but the modifications needed are not difficult and have been described in a private communication by M. Hirsch and C. Pugh. (See also $\left[16\right.$, p. 189].) A second technical point is that $\Phi_{\mu}(x)$ as we have defined it, while smooth in $x$, need not be smooth in the variable $\mu$, so the version of the theorems referred to is not applicable as it stands. However, if one only insists that the resulting manifolds and invariant circles vary continuously in $\mu$, continuity of $\Phi_{\mu}(x)$ in $\mu$ is enough. This requires a careful examination of the proofs since the theorems are not stated in a form convenient for this deduction.

Having found unique invariant circles $c_{\mu}$ for $\Phi_{\mu}$ it follows that $c_{\mu}$ are actually flow invariant since $F_{t}^{\mu}\left(c_{\mu}\right)=F_{t}^{\mu}\left(F_{1}\left(c_{\mu}\right)\right)=F_{1}^{\mu}\left(F_{t}\left(c_{\mu}\right)\right)$ so by uniqueness, $F_{t}^{\mu}\left(c_{\mu}\right)=c_{\mu}$. (We have $F_{t}^{\mu}\left(c_{\mu}\right) \cap\{0\}=\varnothing, \mu>0$ by uniqueness of solutions.) It follows that $c_{\mu}$ are actually the periodic solutions of the system we sought. The attracting nature of $c_{\mu}$ together with the continuation assumption entails that the flow is global in time near these periodic orbits and that initial data near $c_{\mu}$ will approach the periodic solution as $t \rightarrow \infty$.

ACKNOWLEDGEMENTS. The author thanks D. Ruelle for his hospitality 
at I.H.E.S. in Bures-sur-Yvette where this work was begun, N. Kopell, M. Hirsch and C. Pugh for valuable conversations, and J. Glimm for encouragement.

\section{REFERENCES}

1. P. Chernoff and J. Marsden, On continuity and smoothness of group actions, Bull. Amer. Math. Soc. 76 (1970), 1044-1049. MR 42 \# 419.

2. N. N. Bruslinskaja, On the behaviour of the solutions of the equations of hydrodynamics when the Reynolds number passes through a critical value, Dokl. Akad. Nauk SSSR 162 (1965), 731-734 = Soviet Math. Dokl. 6 (1965), 724-728. MR 31 \#6460.

3. D. Ebin and J. Marsden, Groups of diffeomorphisms and the motion of an incompressible fluid, Ann. of Math. (2) 92 (1970), 102-163; II (to appear). MR 42 \#6865.

4. A. Fischer and J. Marsden, The Einstein evolution equations as a first-order quasi-linear symmetric hyperbolic system, Comm. Math. Phys. 28 (1972), 1-38.

5. C. Foias and G. Prodi, Sur le comportement global des solutions non-stationnaires des équations de Navier-Stokes en dimension 2, Rend. Sem. Mat. Univ. Padova 39 (1967), 1-34. MR 36 \#6764.

6. M. Hirsch and C. Pugh, Stable manifolds and hyperbolic sets, Proc. Sympos. Pure Math., vol. 14, Amer. Math. Soc., Providence, R.1., 1970, pp. 133-163.

7. M. Hirsch, C. Pugh and M. Schub, Invariant manifolds, Bull. Amer. Math. Soc. 76 (1970), 1015-1019. (Expanded version to appear).

8. E. Hopf, Abzweigung einer periodischen Lösung eines Differentialsystems, Akad. Wiss. Leipzig 94 (1942), 1-22.

9. G. Iooss, Théorie nonlinéaire de la stabilité des écoulements laminaires dans le cas de "l'échange des stabilités", Thèse, Faculté des Sciences, Paris, 1971; Arch Rational Mech. Anal. 40 (1971), 166-208. MR 42 \# 4074; ibid 47 (1972), 301-329.

10. D. Joseph and D. Sattinger, Bifurcating time periodic solutions and their stability, Arch. Rational Mech. Anal. 45 (1972), 79-109.

11. R. Jost and D. Zehnder, A generalization of the Hopf bifurcation theorem, Helv. Phys. Acta 45 (1972), 258-276.

12. V. Judovich, Example of the generation of secondary stationary or periodic flow when there is a loss of stability of the laminar flow of a viscous incompressible fluid, J. Appl. Math. Mech. 29 (1965), 527-544.

13. J. Marsden, On product formulas for nonlinear semigroups, J. Functional Analysis (to appear).

14.

15. J. Marsden, D. Ebin and A. Fisher, Diffeomorphism groups, hydrodynamics and relativity, Proc. Thirteenth Canad. Math. Congress 1971, pp. 135-279.

16. D. Ruelle and F. Takens, On the nature of turbulence, Comm. Math. Phys. 20 (1971), 167-192; ibid. 23 (1971), 343-344. MR 44 \# 1297.

17. D. Sattinger, Bifurcation of periodic solutions of the Navier-Stokes equations, Arch. Rational Mech. Anal. 41 (1971), 66-80. MR 42 \#7138.

18. - Topics in stability and bifurcation theory, Lecture Notes, University of Mininesota, Minneapolis, Minn., 1972 (mimeographed).

19. I. Segal, Non-linear semi-groups, Ann. of Math. (2) 78 (1963), 339-364. MR 27 \#2879.

Department of Mathematics, University of CAlifornia, Berkeley, California 94720 